\title{
Molecular Diagnosis of Infectious Diseases Using Cytological Specimens
}

Sule Canberk, M.D., M.I.A.C., ${ }^{1}$ Adhemar Longatto-Filho, M.Sc., Ph.D., P.M.I.A.C., ${ }^{2,3,4,5}$ and Fernando Schmitt, M.D., Ph.D., F.I.A.C., ${ }^{6,7 *}$

Pathologists have an important role in the diagnosis of infectious disease (ID). In many cases, a definitive diagnosis can be made using cytopathology alone. However, several ancillary techniques can be used on cytological material to reach a specific diagnosis by identifying the causative agent and consequently defining the management of the patient. This review aims to present the effectiveness of the application of molecular studies on cytological material to diagnose IDs and discuss the advantages and disadvantages of the various molecular techniques according to the type of cytological specimen and the infectious agents. Diagn. Cytopathol. 2016;44:156-164. (C) 2015 Wiley Periodicals, Inc.

Key Words: gynecological cytology; non-gynecological cytology; molecular methods; infectious disease; fine-needle aspiration cytology

\footnotetext{
${ }^{1}$ Department of Pathology-Cytopathology, Haydarpasa Numune Education and Research Hospital, Istanbul, Turkey

${ }^{2}$ Laboratory of Medical Investigation (LIM) 14, Faculty of Medicine, São Paulo University, FMUSP, São Paulo, Brazil

${ }^{3}$ Life and Health Sciences Research Institute (ICVS), School of Health Sciences, University of Minho, Braga, Portugal

${ }^{4}$ ICVS/3B's, PT Government Associate Laboratory, Braga, Portugal

${ }^{5}$ Molecular Oncology Center, Barretos Cancer Hospital, Barretos, São Paulo, Brazil

${ }^{6}$ Department of Pathology and Medicine, Laboratoire National De Sante, Dudelange, Luxembourg

${ }^{7}$ Instituto De Patologia E Imunologia Molecular Da Universidade Do Porto (IPATIMUP) E Faculdade De Medicina Do Porto, Porto, Portugal

*Correspondence to: Fernando Schmitt, Director of the Department of Pathology and Medicine, Laboratoire National de Sante, 1, rue Louis Rech, L-3555 Dudelange, Luxembourg. E-mail: Fernando.SCHMITT@Ins.etat.lu

Conflict of Interest statement: The authors have no Conflict of Interest to declare.

Disclosure of Grants or other Fundings: The authors have no funding to disclose.

Received 13 November 2015; Accepted 13 November 2015

DOI: $10.1002 /$ dc. 23394

Published online 1 December 2015 in Wiley Online Library (wileyonlinelibrary.com).
}

Infectious diseases (ID) are well-acknowledged threats to global human health in the past and at present. Since the first report of Grieg and Gray in 1904, ${ }^{1}$ that stated "the potential of fine-needle aspiration (FNA) in the diagnosis of ID," the role of cytopathology in ID has expanded enormously with the advances in molecular techniques. Cytopathologists are frequently the first to diagnose an ID, and cytopathology material can be used to follow the disease and monitor the response to the treatment. However, the diagnosis of ID and the specific causative agent based solely on the cytomorphology and traditional ancillary techniques has limitations and needs to be correlated with additional techniques including molecular methods.

The integration of molecular methods into the diagnosis of ID using cytology material requires educated personnel, facilities and set work flow designs, but most importantly the methods have to be standardized and performed in collaboration with a clinical microbiologist. It has been shown that costs will be reduced in the longterm by the use of molecular methods compared with conventional methods in the diagnosis of ID: patient outcome are improved, costs are reduced by the use of correct antimicrobials, and hospital stay times will be shortened reducing nosocomial infections. ${ }^{2}$

In the literature, studies have focused on the diagnosis of granulomatous diseases using molecular methods on cytology specimens. This review contains comprehensive, up to date, and systematic information covering the use of molecular techniques to diagnose ID in gynecological and non-gynecological cytological material.

\section{Molecular Methods}

Molecular diagnostics of IDs are based particularly on nucleic acid assay methods. There are three main 


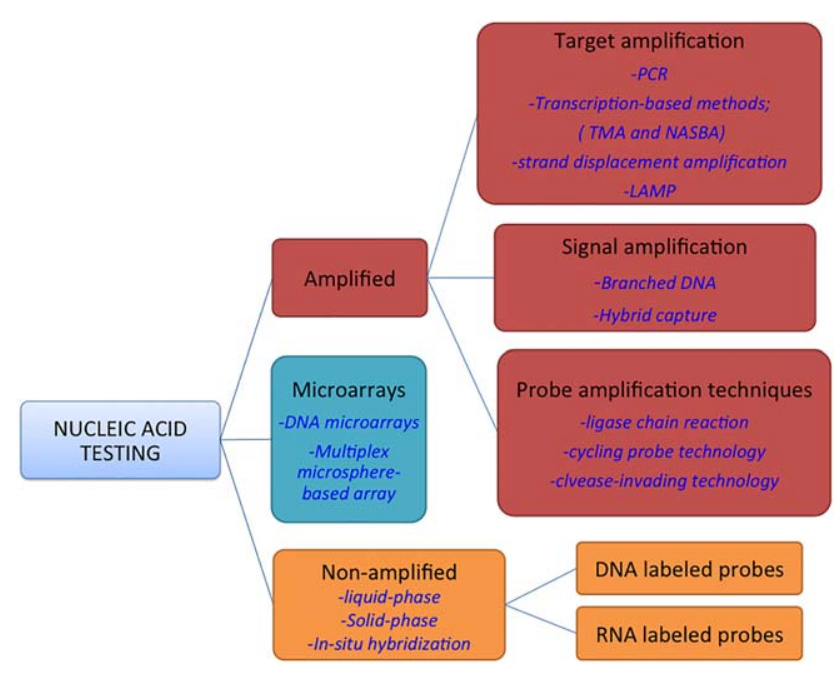

Fig. 1. Nucleic acid testing techniques. [Color figure can be viewed in the online issue, which is available at wileyonlinelibrary.com.]

categories of nucleic acid test: amplified, non-amplified, and microarrays.

\section{Amplified Nucleic Acid Techniques}

Amplified DNA based methods are composed of three subcategories as target, signal, and probe amplification. The polymerase chain reaction (PCR) amplification- and hybridization-based approaches are the most widely used ones in amplified techniques (Fig. 1). ${ }^{3-5}$ Target amplification methods utilize post-amplification analysis where required.

PCR is the most common target amplification method to detect cultured and uncultured bacteria used currently in clinical practice. ${ }^{6}$ Since 1985 many PCR amplification based techniques have been designed for bacterial detection and identification, including: competitive PCR, mostprobable number PCR (MPN-PCR), co-operational PCR, BIO-PCR, nested-PCR, and real-time PCR. Real-time PCR is the most useful and commonly used method because it is faster, has lower contamination risk and higher sensitivity, and has quantitative applications. PCR analysis can be applied directly to fresh material obtained from FNA, to liquid-based cytology materials and even to cells scraped from slides. ${ }^{7}$ DNA is amplified by repeating three major steps, which are denaturation of the DNA template, annealing of oligonucleotide primers, and extension of the primers by DNA polymerase, to produce a copy of the target gene. ${ }^{3}$ To yield high quality DNA is definitely the most critical step.

Unlike PCR, transcription based methods (TBM) and strand displacement amplification (SDA) are isothermal techniques that do not require a thermal cycler. ${ }^{8}$ Nucleic acid sequence-based amplification (NASBA) and transcription-mediated amplification (TMA) are TBM in which the RNA target is reverse transcribed into cDNA and then RNA copies are synthesized with a RNA polymerase. Both TBM and SDA have been used particularly for sexually transmitted diseases (e.g., Chlamydia trachomatis and Neisseria gonorrheae), although false-positive results with this technique have been reported in the literature. 8,9

Signal amplification assays are correlated with the amount of the target sequence in the cytological material. ${ }^{4,8}$ bDNA assays usually are used to detect HCV, HBV, and HIV-1 blood levels and are based on the balance between capture probe bDNA assays and the quantitation of the microorganism. ${ }^{3,8}$ Loop-mediated isothermal amplification (LAMP) is a one-step technique which is very similar to SDA and has been developed for cytomegalovirus (CMV), herpes simplex virus (HSV), varicella zoster virus (VZV), BK virus, and human papillomavirus (HPV). ${ }^{8}$

In the last two decades, hybrid capture has become a popular technique applicable to gynecological cytology specimens. Briefly, the method entails the reaction between DNA-RNA hybrids and anti-hybrid antibodies, with a luminometer measuring the light emitted. ${ }^{3,8}$ Chlamydia trachomatis, Neisseria gonorrheae, CMV, and particularly HPV are the common agents detected by this method. ${ }^{3}$

The most important benefit of amplification methods is the requirement for low target copy numbers within a direct specimen. On the other hand, contamination is the main problem of the amplified techniques when compared with microarrays and non-amplified methods. ${ }^{3,10}$ Also, the evaluation of the sensitivity of each specimen type and the setting up of a number of positive and negative controls for each diagnostic run are vital for rendering standardization, which is sine qua non for molecular studies. ${ }^{11,12}$

\section{Microarrays}

Microarrays, multiplex nucleic acid amplification techniques, and mass spectrometry are multiparameter assay techniques that provides rapid diagnoses with a decreased contamination risk, low cost, high sensitivity/specificity, and rapid kinetics through the use of a closed-tube systems. ${ }^{5,8}$

\section{Non-Amplified Nucleic Acid Techniques}

The well-known format is probe hybridization comprised of liquid phase, solid phase, and in situ hybridization. Different species can be detected by in-situ hybridization in the one clinical sample in the same run through use of multiple probes labeled with different fluorescent dyes. ${ }^{9}$ It is a very commonly used and rapid technique with the four "S" advantages: safe, simple, specific, and sensitive in detecting and identifying the microorganisms directly from the clinical specimen such as a smear. The presence of unbound probes and the nonspecific binding of the probe to non-target microorganisms may give false results with this technique. ${ }^{9}$ 


\section{Applications in Non-Gynecological Cytology Exfoliative Cytology}

Bronchoalveolar Lavage and Sputum. Community acquired pneumonia (CAP) is the major cause of death due to ID in the United States and worldwide. ${ }^{13}$ Due to the high contamination risk of upper airway flora, cytology specimens may show diagnostic limitations. As the most common agent of CAP, Streptococcus pneumonia is a fastidious bacterium which needs special environmental and nutritional conditions to culture, and so molecular methods are recommended for its detection. The concentration of the bacteria in the cytological specimen is a significant step in deciding whether it is a pathogen and which is the appropriate molecular method to diagnose the CAP. Streptococcus pneumoniae, Mycoplasma pneumonia, Staphylococcus aureus, and Chlamydia pneumoniae among others, should be detected at a certain concentration before they are considered a pathogen. However, even trace amounts of Mycobacterium tuberculosis, Bacillus anthracis, Legionella, endemic fungi, Yersinia pestis, and Francisella tularensis are assumed pathogenic regardless of the concentration. Real-time PCR is the best method for use in this situation because is also quantitative.

Invasive aspergillosis (IA) is a significant cause of death in cancer patients. The concentration threshold is also important in the diagnosis of aspergillosis, and a quantitative PCR (real-time PCR) is recommended to differentiate colonization from infection. Sun et al. reported the sensitivity of $95 \%$ and specificity of $95 \%$ in the diagnosis of Aspergillus species with PCR. ${ }^{14}$

Paracoccidioides brasiliensis can cause a systemic mycosis agent that is not limited to immune-compromised patients. It is only seen in Latin America and is accepted as endemic particularly in Brasil. Histoplasma spp. and Coccidioides immitis can be misinterpreted as paracoccidioidomycosis. LAMP was suggested as a highly specific and sensitive method to identify the target $P$. brasiliensis P43 gene in sputa. ${ }^{15-17}$ Coccidioides immitis and Blastomyces dermatitidis are other examples of systemic fungal infections that can be misdiagnosed clinically and morphologically, and specifically diagnosed on DNA probes specific for unique RNA sequences.

Mycobacterium africanum and $M$. canetti mainly seen in African countries cause human tuberculosis and share almost identical genomes, called "the mycobacterium tuberculosis complex organisms" (MTC), along with Mycobacterium tuberculosis and Mycobacterium bovis which are the most common members of this group in all developing countries. However, it is almost impossible to differentiate these mycobacteria using conventional methods. Mycobacterium bovis is an increasing threat particu- larly in Asia, Latin America and the HIV-infected patient group and can cause pulmonary and extrapulmonary tuberculosis in humans. ${ }^{18}$ Bakshi et al. showed that it is possible to distinguish $M$. bovis from $M$. tuberculosis using a multiplex PCR based on the absence of a $12.7 \mathrm{~kb}$ fragment in $M$. bovis which is present in M. tuberculosis. ${ }^{18}$ Chen et al. using the microsphere based multiplex assay in human sputum reported high identification rates to separate $M$. tuberculosis and M. bovis species, $98.9 \%$ and $91.9 \%$, respectively. ${ }^{19}$ Neonakis et al. detected $M$. tuberculosis with $88.2 \%$ sensitivity and $99 \%$ specificity by using LAMP in clinical specimens. As in the study of Iwamoto et al. and Aryan et al. LAMP has been established as a sensitive, rapid, and low-cost method for typical and atypical mycobacteria including $M$. tuberculosis and $M$. bovis (including $M$. bovis BCG), and the less common species $M$. africanum, $M$. microti, $M$. canetti, $M$. caprae, and $M$. pinnipedii in clinical samples. ${ }^{20-22}$ There are other applications of real-time PCR to detect infectious agents in respiratory cytological samples that are discussed further in the section on FNA.

Effusions. Chronic pericardial or pleural non-malignant effusions need to be analyzed for possible infection, the most common of which is tuberculosis. The benefits of PCR and usefulness of DNA PCR in diagnosing tuberculosis are well documented in the literature. ${ }^{23,24}$

Urine. BKV and John Cunningham virus (JCV) are the human polyoma viruses that are responsible of primary infection in almost $80 \%$ of the healthy population. After a latency period in the genitourinary tract including kidneys, viruses may be reactivated in immunodeficiency conditions such as HIV-infected or kidney transplant patients. Specific cytomorphologic findings, including Decoy cells, may be seen in $4 \%$ of urine samples. The studies based on PCR and particularly nested-PCR (diagnostic accuracy 56\%) from urine specimens were reported as the most sensitive methods to detect polyomavirus infections and identify as the causative agent BKV or JCV. ${ }^{25-27}$

Cerebrospinal Fluid $(C S F)$. PCR, NASBA, and DNA branched assays have been used successfully to detect both RNA and DNA viruses in CSF for diagnosing viral encephalitis. The sensitivity of these techniques depends on the amount of the CSF sample ranging from 30 to 100-200 mL. Real time and nested PCR have high utility with the various primers for pan-herpesvirus assays in the detection of HSV-1,HSV-2, VZV, CMV and Epstein-Barr virus $(E B V)$, and human herpesvirus-6 (HHV-6) in various combinations. ${ }^{28-30}$ These assays are proven as clinically useful in the assessment of HIV patients presenting with a CNS disease, since multiple herpes viruses are capable of causing neurologic symptoms in this subset of patients. $H S V-1$ and $-2, E B V$, and $V Z V$ are the most common agents of sporadic viral encephalitis and Multiplex PCR 
assays may be the best method to detect these agents in a single CSF specimen. ${ }^{28}$

\section{Fine-Needle Aspiration Cytology (FNAC)}

Lymph Node. It is commonly assumed that M. tuberculosis is the commonest cause of lymphadenitis, but PCR studies have shown that Bartonella henselae is the most common agent in both adults and children, presenting mostly as Cat Scratch Disease (CSD), although this is dependent on the population studied. ${ }^{31}$ Avidor et al. showed that FNAC specimens for cytologic evaluation and PCR testing has a 94\% sensitivity for the diagnosis of CSD, while culture, Warthin Starry silver impregnation stain and other stains offer low sensitivity. ${ }^{32}$ Fenollar et al. emphasized that sequencing and hybridization techniques particularly are successful in identifying the species of Bartonella infection with visceral involvement such as bacillary angiomatosis, peliosis hepatitis, and CSD. $^{6}$

Extrapulmonary tuberculosis (EPTBC) is a widespread form of tuberculosis and most commonly presents in head and neck lymph nodes. FNAC is the first choice for the diagnosis, but early in the infection and in the absence of typical granulomas and or caseous necrosis and the frequent absence of acid fast bacilli the diagnosis may not be able to be made, and the distinction from other granulomatous entities including atypical mycobacteria on morphology alone may not be possible. Even the classical cytomorphology of EPTBC has lower sensitivity or specificity with FNAC associated with the traditional methods. The sensitivity of the Ziehl-Neelsen stain is reported as $20 \%$ up to $43 \%$ for diagnosing and monitoring the treatment of EPTBC. ${ }^{33}$ Culture is regarded as the "gold standard" but needs 6-12 weeks and should be performed in a biosafety level 3 faculty. ${ }^{34}$ Unfortunately, time is the most important factor in the diagnosis of an ID, particularly in immunocompromised conditions such as AIDS. In the study of Goel et al. nucleic acid testing (NAA) positivity for mycobacterial infection was reported in $72 \%-73 \%$ in fresh aspirates, regardless of the presence or absence of AFB. ${ }^{34}$ Based on the study of Pruhit et al. PCR provided high sensitivity, specificity, and positive and negative predictive values $(85 \%, 95 \%, 96 \%$, and $59 \%$, respectively) by using unstained air-dried cytology smears in 98 cases for the early and specific diagnosis of EPTBC. In the same study, Ziehl-Neelsen stain and culture was able to detect mycobacteria in $15.3 \%-24.4 \%$ of cases. $^{35}$ Due to the differences in treatment of typical, atypical mycobacteriosis, and the other granulomatous conditions, it is crucial to detect and identify bacteria with a high diagnostic accuracy.

Toxoplasma gondii may cause a suppurative lymphadenitis localized in posterior cervical lymph nodes. FNAC is very useful to distinguish this infection from malignant lymphoma and PCR can identify the protozoon.

Biological and clinical differences between HPVrelated and HPV non-related head and neck squamous cell carcinoma (HNSCC) effect the target therapy, response, and or resistance to the therapy as well as the prognosis. Differentiating HPV related from non-related SCC based solely on morphology is impossible and the molecular test has become best practice over the past few years. $^{36,37}$ HPV16 is the commonest serotype found in $75 \%-92 \%$ of HPV-related HNSCC. Methods for detecting HPV use different targets such as, HPV DNA, HPV RNA, E6 E7 viral oncoproteins, and P16 as cellular protein. PCR based amplifications and DNA in situ hybridizations are used routinely, and the latter is very practical and allows visualization of the cells with high risk (HR) HPV DNA making the method highly specific. On the other hand, PCR based amplification methods (discussed in the gynecology part of this review) are much more sensitive and specific, but less practical. Although PCR, DNA in situ hybridization, and hybrid capture II and the Cervista technique (able to detect 14 sero-types) show variability in sensitivity and specificity, these methods have been successfully used to detect HR HPV in cytological material, even in the necrosis found in FNA material of Head and Neck metastases. ${ }^{36-39}$ In the prospective study of Baldassari et al. HR-HPV detection-genotyping performed on the FNA material from metastatic SCC in lymph nodes using the Roche Cobas 4800 system showed a sensitivity of $90 \%$ which is greater than studies using hybrid capture II and the Cervista technique which usually give high specificity but low sensitivity. ${ }^{40}$

Lung. The opportunistic infections in immune compromised patients including HIV positive, cancer, and posttransplant patients, may mimic lung malignancies. Pneumocystis jiroveci is a major pathogen and can involve extrapulmonary sites including liver, spleen, skin, and pleural effusions. Specific diagnosis is based mainly on respiratory cytology materials. Bronchiolo-alveolar lavage (BAL) has a diagnostic yield of $97 \%-100 \%$, superior to sputum with a specificity of $100 \%$ and sensitivity of $55 \%$ in the diagnosis of $P$. jiroveci. ${ }^{41}$ Invasive procedures such as BAL and transthoracic FNAC may yield better diagnoses. Quantitative PCR (RT-PCR) and nested PCR are the widespread techniques now used in the diagnosis of pneumocystis pneumonia, and RT-PCR can differentiate colonization from infection in non-HIV patients. Both techniques have shown high specificity and sensitivity rates in many articles. ${ }^{42-44}$

Histoplasma capsulatum, Cryptococcus neoformans, and Cryptococcus gattii may cause cavitating or solid lesions often large in size that can be misdiagnosed is lung malignancies. Mycobacterial infection (tuberculosis) of respiratory samples may be heralded by finding 
Table I. Details of Most Frequently Used Commercially Available Tests for HPV Identification

\begin{tabular}{|c|c|c|c|}
\hline Test & Origin & Target & Characteristics \\
\hline Hybrid Capture 2 & Qiagen, Hilden, Germany & DNA & $\begin{array}{l}13 \text { hrHPV types (Hybrid DNA:RNA): } 16,18,31,33,35,39,45 \text {, } \\
51,52,56,58,59 \text {, and } 68\end{array}$ \\
\hline Cobas 4800 HPV Test & Roche, Pleasanton, The United States & DNA & $\begin{array}{l}14 \text { hrHPV types (PCR technology) and HPV16 and HPV } 18 \\
\text { identification, distinctly; and } 31,33,35,39,45,51,52,56,58 \text {, } \\
59,66 \text {, and } 68\end{array}$ \\
\hline Abbot HPV Assay & Abbot, Weisbaden, Germany & DNA & $\begin{array}{l}\text { Two hrHPV types individually (RT PCR): } 16 \text {, and } 18 \text {; and also } \\
12 \text { high risk genotypes: } 31,33,35,39,45,51,52,56,58,59 \text {, } \\
66,68\end{array}$ \\
\hline BD HPV Test & BD, Sparks, The United States & DNA & $\begin{array}{l}\text { Six hrHPV types individually (RT PCR): } 16,18,45,31,51 \text { and } \\
52 \text { and three groups of hrHPV: } 33 \text { and } 58 ; 35,39 \text {, and } 68 \text {; and } \\
56,59 \text { and } 66 .\end{array}$ \\
\hline PreTect HPV-Proofer & Hologic, Klokkarstua, Norway & RNA & $\begin{array}{l}\text { E6/E7 mRNA-based test for oncogenic types } 16,18,31,33 \text {, and } \\
45 .\end{array}$ \\
\hline APTIMA & $\begin{array}{l}\text { Gen-Probe Incorporated, } \\
\text { San Diego, The United States }\end{array}$ & RNA & $\begin{array}{l}\text { E6/E7 mRNA of } 14 \text { high-risk HPV types: } 16,18,31,33,35,39 \text {, } \\
\text { 45, 51, 52, 56, 58,59, 66, and 68; with HPV } 16 \text { and } 18 / 45 \\
\text { genotyping. }\end{array}$ \\
\hline
\end{tabular}

granulomatous inflammation with or without necrosis. FNA material is suitable for both traditional and molecular diagnostic methods. A combination of acid fast stain with PCR has high sensitivity $(84 \%)$ and specificity $(100 \%){ }^{41}$ Many studies have focused particularly on realtime and nested-PCR and sputum and BAL samples a well as FNA. ${ }^{4,46}$ Viral pulmonary infections, such as CMV, Respiratory Syncytial Virus, and Herpes simplex, can also be confirmed using molecular tests on cytological samples.

Skin. Buruli ulcer is an ID of skin presenting with a painless nodule, a firm plaque or an edematous lesion in children aged 15 years or younger and is caused by Mycobacterium ulcerans. It is common in over 30 countries worldwide, particularly humid tropical areas in West Africa. ${ }^{47}$

Edyani et al. proved that FNAC is an effective diagnostic tool using the PCR technique, and that higher diagnostic accuracy was achieved using samples from nonulcerated compared with ulcerated lesions. ${ }^{48}$ The application of PCR to the FNAC samples utilizing 21-gauge needles yielded $86 \%$ sensitivity compared with culture with $44 \%$ and microscopy with $26 \%$ as demonstrated by Phillips et al. ${ }^{47}$

Real-time PCR has been suggested for detection, quantification and identification of Leishmania species in clinical samples. ${ }^{49}$ Traditional methods yield $17 \%$ positivity in the patients with mucosal leishmaniasis while PCR achieved $71 \%$ positivity in the study of Tavares. ${ }^{49}$

\section{Applications in Gynecological Cytology}

The introduction of liquid-based cytology (LBC) in routine gynecological cytology produced better quality preparations for cytomorphology and the potential to perform complementary molecular assessment on residual material, particularly molecular tests for HR-HPV detection in cervical cancer prevention programs. The HR-HPV test increases the sensitivity of the "Pap test," and can achieve an acceptable specificity when used alone. ${ }^{50}$

\section{HPV Test and Cervical Cancer Screening}

The introduction of HPV test for primary cervical cancer screening has been contentious, but recent studies suggest that HPV tests can be used for this purpose. ${ }^{51}$ The rational that supports the use of HPV testing in cervical cancer prevention is that the presence of HPV is necessary for cervical cancer development, ${ }^{52}$ and therefore a negative HPV test excludes the development of highgrade lesions. The age to start HPV test screening has been debated but it should be set so as to avoid unnecessary tests while still reaching acceptable levels in detecting $\geq$ CIN2 lesions. Women aged $\geq 30$ year's benefit the most with HPV-based screening. It is also critical to validate the HPV tests considered for use, which must have a sensitivity for $\geq$ CIN2detection equal or $90 \%$ and a specificity not $<98 \%$. ${ }^{53}$

A plethora of HPV tests have been developed in recent years, and choice of a specific test depends on a number of requisites that range from high performance indexes, to the workload capacity, local commercial concerns and to equipment maintenance, logistical issues, training, and cost. The majority of the tests target DNA as does the doyen Hybrid Capture technology; while others target RNA, for example, the NorChip PreTect HPV-Proofer. The important issue, however, is to identify hrHPV with high clinical sensitivity and acceptable specificity. Cuzick and colleagues ${ }^{54}$ compared the performance of six hrHPV tests commercially available: Hybrid Capture 2 (Qiagen, Germany), Cobas 4800 HPV Test (Roche, The United States), Abbot RealTime High Risk HPV Assay (Abbot, Germany), BD HPV Test (BD Diagnostic, The United States), PreTect HPV-Proofer (NorChip, Norway), and APTIMA (Gen-Probe, The United States). Details on the HPV tests system are depicted in Table I. The authors 
Table II. Details of Most Frequently Used Commercially Available Tests for Other Genital Microorganisms

\begin{tabular}{|c|c|c|c|}
\hline Test & Origin & Target & Characteristics \\
\hline $\begin{array}{l}\mathrm{COBAS}^{\circledR} \text { AMPLICOR } \\
\text { (CT/NG) Tes }\end{array}$ & $\begin{array}{l}\text { Roche, Pleasanton, } \\
\text { The United States }\end{array}$ & DNA & $\begin{array}{l}\text { PCR amplification technique for the detection of Chlamydia trachomatis } \\
\text { and/or Neisseria gonorrhoeae }\end{array}$ \\
\hline BD ProbeTec ET & BD, Sparks, The United States & DNA & $\begin{array}{l}\text { Strand Displacement Amplification (SDA) technology for C. trachoma- } \\
\text { tis, N. gonorrhoeae, or both. }\end{array}$ \\
\hline $\begin{array}{l}\text { Qiagen HC2 CT/GC } \\
\text { DNA Test }\end{array}$ & Qiagen, Hilden, Germany & DNA & $\begin{array}{l}\text { Chemiluminescent signal-amplified nucleic acid hybridization for Chla- } \\
\text { mydia trachomatis and/or Neisseria gonorrhoeae }\end{array}$ \\
\hline $\begin{array}{l}\text { Abbott RealTime } \\
\text { CT/NG assay }\end{array}$ & Abbot, Weisbaden, Germany & DNA & Real-time PCR Chlamydia trachomatis and/or Neisseria gonorrhoeae. \\
\hline АРТIMA COMBO 2 & $\begin{array}{l}\text { (Hologic Gen-Probe } \\
\text { Incorporated, San Diego, } \\
\text { The United States ) }\end{array}$ & RNA & $\begin{array}{l}\text { PCR amplification technique for the detection of Chlamydia trachomatis } \\
\text { and/or Neisseria gonorrhoeae }\end{array}$ \\
\hline
\end{tabular}

summarized these six HPV assay methods and observed the following sensitivity, specificity, and PPV for CIN2 results tests for $\mathrm{HC} 2$ as $97.5 \%, 85.4 \%$, and $4.3 \%$, Cobas HPV as $97.5 \%, 84.5 \%$, and 4.1 and APTIMA HPV as $97.5 \%, 90.2 \%$, and $6.3 \% .^{54}$ Recently, Nolte et al. designed a comparison study of performance characteristics of Aptima and Cervista HPV tests: Aptima HPV test was found to be as Cervista, however, specificity was higher compare to Cervista in the detection of hrHPV in cervical cytology specimens. ${ }^{55}$

Most studies have shown that the RNA based assay APTIMA system has high specificity and sensitivity compared with the other assays. ${ }^{56}$

The performance of the DNA-based assays was quite similar with a slightly lower positivity rate for the Abbott system in the group of women who had tested negative in the Pap test. Both RNA-based tests had lower performances in HPV detection in comparison with the DNA tests, but NorChip had significantly lower performance even when compared with the APTIMA test. All cases categorized as CIN3 were positive in all tests, with the exception of one case which tested negative in the Abbott assay and five negative in the NorChip test. ${ }^{54}$ However, the main aim is to show not only transient infections which in most cases are self-limited but also integrated hrHPV viral infections which lead to squamous dysplasia and neoplasia. ${ }^{56}$ APTIMA demonstrates the overexpression with the E6 and E7 mRNA of hrHPV. The viral genes E6 and E7 may inactivate tumor suppressor genes (P53 and pRB) during the integration into the host genome and activate the carcinogenesis process. ${ }^{57}$ In Sauter et al. the authors reported $21 \%$ and $90 \%$ decreased colposcopy referral and QNS (quantity not sufficient) rates, respectively, with APTIMA compared with HC2. ${ }^{56}$ APTIMA is the most well-known RNA based detection assay that detects the $16 \mathrm{hrHPV}$ subtypes but cannot differentiate specifically between them, ${ }^{56}$ and the clinical evaluation of APTIMA HPV RNA (CLEAR) trial showed other limitations including cross-reactivity with the lowrisk HPV subtypes 26, 67, 70, 82, and crosscontamination of samples $^{58}$ (US Food and DrugAdminis- tration. APTIMAVR HPV Assay Labeling. accessdata.fda.gov/cdrh_docs/pdf10/P100042c.pdf. Accessed January 22, 2014).

DNA-based assays include HC2, Cervista, and Cobas. $\mathrm{HC} 2$ and Cervista utilize signal amplification methods. A large amount of important data on HC2 was collected in the ALTS study (atypical squamous cells of undetermined significance [ASCUS]/low-grade squamous intraepithelial lesion [LSIL] Triage Study). ${ }^{59}$ However, HC2 does not have an internal control and may show cross-reaction with some low risk HPV subtypes representing limitations despite its high clinical sensitivity. ${ }^{58}$ Cervista uses the "Invader chemistry" (Hologic) and targets the HPV L1 gene. Cervista has an internal control with the patient specimen, but some studies have shown that it has distinctive limitations during processing with the glacial acetic acid. ${ }^{60}$ Cobas is a PCR amplification test studied in 47,000 women in the ATHENA trial (Addressing the Need for Advanced HPV Diagnostics) which demonstrated excellent performance. ${ }^{61}$ The U.S. Food and Drug Administration $^{62}$ approved the Cobas 4,800 HPV Test for use in primary screening in women $\geq 25$ years. As the first prospective U.S. screening study, the ATHENA ${ }^{63}$ end-results have very recently been published and it has been incorporated into current guidelines (http://dx.doi. org/10.1016/j.ygyno.2014.11.076.). This recommendation is important because this is a paradigm change for cervical cancer prevention. The adoption of molecular testing for primary cervical cancer screening has been seriously considered by many public authorities in European Countries, ${ }^{64,65}$ where cytology-based screening has been successfully implemented.

Larsson et al. performed the extended genotyping approach with Anyplex II HPV28, which detects 28 genotypes and the human gene beta-globulin HBB in two multiplex reactions, and CLART HPV2 (Genomica), which detects 35 genotypes and the human gene CTFR in one reaction targeting the $\mathrm{L} 1$ region of the virus, using archival clinical samples and found both were suitable alternative methods even in the presence of minor intraassay differences. ${ }^{66}$ 
Finally, the hrHPV assay test can be used on residual liquid media material importantly in patients, who have had cervical conization for high grade lesions, ${ }^{67}$ to determine hrHPV positive patients who are at risk of recurrence after surgery. The hrHPV test can also be used as a reflex test in cases of cytological atypia of undetermined significance (ASC-US) ${ }^{68}$ to improve the detection of high grade lesions. Liquid media also can be used in the methylation marker test (CADM1/MAL methylation analysis) as a triage test in hrHPV positive women in the presence of CIN2 and CIN3. ${ }^{69}$

\section{The Use of Liquid Medium for Other Genital Infections}

Many of the microorganisms usually found in cervicalvaginal region are also detectable in liquid media using different molecular tests, which are easy to perform and generate results generally comparable to the traditional, but generally time consuming, assays such as vaginal sample cultures or conventional PCR. One of the most interesting advances are the very robust Chlamydia and gonorrhea organisms tests, performed in a "combo fashion" system (Table II).

Liquid-based cytology medium samples can be used to preserve urine similarly to vaginal material for various tests including the successful identification of the Mycoplasma genitalium, Mycoplasma hominis, Ureaplasma parvum, and Ureaplasma urealyticum, in female and male patients. ${ }^{70}$ Importantly, in house PCR molecular methods applied to the residual material from liquid-based cytology medium have efficiently recognized Herpes simplex virus and Cytomegalovirus. ${ }^{71,72}$

\section{Closing Remarks}

The use of molecular methods on cytological material is a milestone in the specific diagnosis of the ID. The integration of nucleic acid testing methods with cytopathology provides improved diagnostic protocols and in some cases a correct diagnosis more rapidly for life saving treatment. However, it is necessary to systematically assess "which" test to utilize, "where" to use it, and "how" to integrate the nucleic acid testing methods with the cytomorphological diagnosis to maximize the diagnostic potential and cost benefit in the challenging diagnosis of it is. In addition to the classical cytology smear preparation, the introduction of liquid-based cytology in both gynecological and non-gynecological investigations has opened up new horizons in terms of ID diagnoses, primary screening options, and post-treatment follow-up methods, demonstrating clear advantages for clinicians and patients.

\section{References}

1. Greig ED, Gray AC. Note on the lymphatic glands in sleeping sickness. Br Med J 1904;1:1252.

2. Krishnamurthy S. Applications of molecular techniques to fineneedle aspiration biopsy. Cancer 2007;111:106-122.

3. Muldrew KL. Molecular diagnostics of infectious diseases. Curr Opin Pediatr 2009;21:102-111.

4. Cobo F, Talavera P, Concha A. Review article: Relationship of human papillomavirus with papillary squamous cell carcinoma of the upper aerodigestive tract: A review. Int J Surg Pathol 2008;16: 127-136.

5. Weile J, Knabbe C. Current applications and future trends of molecular diagnostics in clinical bacteriology. Anal Bioanal Chem 2009;394:731-742.

6. Fenollar F, Raoult D. Molecular genetic methods for the diagnosis of fastidious microorganisms. Apmis 2004;112:785-807.

7. Schmitt F, Barroca H. Role of ancillary studies in fine-needle aspiration from selected tumors. Cancer Cytopathol 2012;120:145-160.

8. Cobo F. Application of molecular diagnostic techniques for viral testing. Open Virol J 2012;6:104-114.

9. Barken KB, Haagensen JA, Tolker-Nielsen T. Advances in nucleic acid-based diagnostics of bacterial infections. Clin Chim Acta 2007;384:1-11.

10. Borst A, Box AT, Fluit AC. False-positive results and contamination in nucleic acid amplification assays: Suggestions for a prevent and destroy strategy. Eur J Clin Microbiol Infect Dis 2004;23:289299.

11. Millar BC, Xu J, Moore JE. Molecular diagnostics of medically important bacterial infections. Curr Issues Mol Biol 2007;9:21-39.

12. Albuquerque P, Mendes MV, Santos CL, et al. DNA signaturebased approaches for bacterial detection and identification. Sci Total Environ 2009;407:3641-3651.

13. Bartlett JG. Diagnostic tests for agents of community-acquired pneumonia. Clin Infect Dis 2011;52:S296-S304.

14. Sun W, Wang K, Gao W, et al. Evaluation of PCR on bronchoalveolar lavage fluid for diagnosis of invasive aspergillosis: A bivariate metaanalysis and systematic review. PLoS One 2011;6:e28467.

15. Endo S, Komori T, Ricci G, et al. Detection of gp43 of Paracoccidioides brasiliensis by the loop-mediated isothermal amplification (LAMP) method. FEMS Microbiol Lett 2004;234:93-97.

16. San-Blas G, Niño-Vega G, Barreto L, et al. Primers for clinical detection of Paracoccidioides brasiliensis. J Clin Microbiol 2005; 43:4255-4257.

17. San-Blas G, Niño-Vega G. Paracoccidioides brasiliensis: Chemical and molecular tools for research on cell walls, antifungals, diagnosis, taxonomy. Mycopathologia 2008;165:183-195.

18. Bakshi CS, Singh VP, Malik M, et al. Polymerase chain reaction amplification of $16 \mathrm{~S}-23 \mathrm{~S}$ spacer region for rapid identification of Salmonella serovars. Acta Vet Hung 2002;50:161-166.

19. Chen R, Bi Y, Yang G, et al. Development of a fluorescent microsphere-based multiplex assay for simultaneous rapid detection of Mycobacterium tuberculosis complex and differentiation of $\mathrm{M}$. tuberculosis and M. bovis in clinical samples. Diagn Mol Pathol 2010;19:172-179.

20. Iwamoto $\mathrm{T}$, Sonobe $\mathrm{T}$, Hayashi K. Loop-mediated isothermal amplification for direct detection of Mycobacterium tuberculosis complex, M. avium, and M. intracellulare in sputum samples. J Clin Microbiol 2003;41:2616-2622.

21. Neonakis IK, Spandidos DA, Petinaki E. Use of loop-mediated isothermal amplification of DNA for the rapid detection of Mycobacterium tuberculosis in clinical specimens. Eur J Clin Microbiol Infect Dis 2011;30:937-942.

22. Aryan E, Makvandi M, Farajzadeh A, et al. A novel and more sensitive loop-mediated isothermal amplification assay targeting 
IS6110 for detection of Mycobacterium tuberculosis complex. Microbiol Res 2010;165:211-220.

23. Prakash KC. Tuberculous peritonitis. Perit Dial Int 1999;19:S283S285.

24. Levy PY, Fournier PE, Charrel R, et al. Molecular analysis of pericardial fluid: A 7-year experience. Eur Heart J 2006;27:1942-1946.

25. Boldorini R, Zorini EO, Viganò $\mathrm{P}$, et al. Cytologic and biomolecular diagnosis of polyomavirus infection in urine specimens of HIVpositive patients. Acta Cytol 2000;44:205-210.

26. Boldorini R, Brustia M, Veggiani C, et al. Periodic assessment of urine and serum by cytology and molecular biology as a diagnostic tool for BK virus nephropathy in renal transplant patients. Acta Cytol 2005;49:235-243.

27. Herawi M, Parwani AV, Chan T, et al. Polyoma virus-associated cellular changes in the urine and bladder biopsy samples: A cytohistologic correlation. Am J Surg Pathol 2006;30:345-350.

28. Debiasi RL, Tyler KL. Molecular methods for diagnosis of viral encephalitis. Clin Microbiol Rev 2004;17:903-925.

29. Bouquillon C, Dewilde A, Andreoletti L, et al. Simultaneous detection of 6 human herpesviruses in cerebrospinal fluid and aqueous fluid by a single PCR using stair primers. J Med Virol 2000;62: 349-353.

30. Calvario A, Bozzi A, Scarasciulli M, et al. Herpes Consensus PCR test: A useful diagnostic approach to the screening of viral diseases of the central nervous system. J Clin Virol 2002;25:S71-S78.

31. Angelakis E, Roux V, Raoult D, et al. Real-time PCR strategy and detection of bacterial agents of lymphadenitis. Eur J Clin Microbiol Infect Dis 2009;28:1363-1368.

32. Avidor B, Varon M, Marmor S, et al. DNA amplification for the diagnosis of cat-scratch disease in small-quantity clinical specimens. Am J Clin Pathol 2001;115:900-909.

33. Derese Y, Hailu E, Assefa T, et al. Comparison of PCR with standard culture of fine needle aspiration samples in the diagnosis of tuberculosis lymphadenitis. J Infect Dev Ctries 2012;6:53-57.

34. Goel MM, Budhwar P, Goel M, et al. Nucleic acid amplification of Mycobacterium tuberculosis complex DNA from archival fine needle aspiration smear scrapings vs. fresh fine needle aspirates of tuberculous lymphadenitis. Acta Cytol 2006;50:393-397.

35. Purohit MR, Mustafa T, Sviland L. Detection of Mycobacterium tuberculosis by polymerase chain reaction with DNA eluted from aspirate smears of tuberculous lymphadenitis. Diagn Mol Pathol 2008;17:174-178.

36. Holmes BJ, Westra WH. The expanding role of cytopathology in the diagnosis of HPV-related squamous cell carcinoma of the head and neck. Diagn Cytopathol 2014;42:85-93.

37. Jarboe EA, Hunt JP, Layfield LJ. Cytomorphologic diagnosis and HPV testing of metastatic and primary oropharyngeal squamous cell carcinomas: A review and summary of the literature. Diagn Cytopathol 2012;40:491-497.

38. Hunt JL. New applications for old assays and the importance of validation. Cancer Cytopathol 2012;120:3-6.

39. Faquin WC. Human papillomavirus (HPV) assays for testing fineneedle aspiration specimens in patients with head and neck squamous cell carcinoma. Cancer Cytopathol 2014;122:92-95.

40. Baldassarri R, Aronberg R, Levi AW, et al. Detection and genotype of high-risk human papillomavirus in fine-needle aspirates of patients with metastatic squamous cell carcinoma is helpful in determining tumor origin. Am J Clin Pathol 2015;143:694-700.

41. Lang TU, Khalbuss WE, Monaco SE, et al. Review of HIV-Related Cytopathology. Patholog Res Int 2011;2011:256083

42. Hauser PM, Bille J, Lass-Flörl C, et al. Multicenter, prospective clinical evaluation of respiratory samples from subjects at risk for Pneumocystis jirovecii infection by use of a commercial real-time PCR assay. J Clin Microbiol 2011;49:1872-1878.
43. Gupta R, Mirdha BR, Guleria R, et al. Improved detection of Pneumocystis jirovecii infection in a tertiary care reference hospital in India. Scand J Infect Dis 2007;39:571-576.

44. Botterel F, Cabaret O, Foulet F, et al. Clinical significance of quantifying Pneumocystis jirovecii DNA by using real-time PCR in bronchoalveolar lavage fluid from immunocompromised patients. J Clin Microbiol 2012;50:227-231.

45. Tiwari V, Jain A, Verma RK. Application of enzyme amplified mycobacterial DNA detection in the diagnosis of pulmonary and extra-pulmonary tuberculosis. Indian J Med Res 2003;118:224-228.

46. Mehta PK, Kalra M, Khuller GK, et al. Development of an ultrasensitive polymerase chain reaction-amplified immunoassay based on mycobacterial RD antigens: Implications for the serodiagnosis of tuberculosis. Diagn Microbiol Infect Dis 2012;72:166-174.

47. Phillips RO, Sarfo FS, Osei-Sarpong F, et al. Sensitivity of PCR targeting Mycobacterium ulcerans by use of fine-needle aspirates for diagnosis of Buruli ulcer. J Clin Microbiol 2009;47:924-926.

48. Eddyani M, Fraga AG, Schmitt F, et al. Fine-needle aspiration, an efficient sampling technique for bacteriological diagnosis of nonulcerative Buruli ulcer. J Clin Microbiol 2009;47:1700-1704.

49. Tavares CA, Fernandes AP, Melo MN. Molecular diagnosis of leishmaniasis. Expert Rev Mol Diagn 2003;3:657-667.

50. Somoza AD, Aly FZ. Utility of molecular tests in cytopathology. Cytojournal 2014;11:5.

51. Schiffman M, Wentzensen N, Wacholder S, et al. Human papillomavirus testing in the prevention of cervical cancer. J Natl Cancer Inst 2011;103:368-383.

52. Walboomers JM, Jacobs MV, Manos MM, et al. Human papillomavirus is a necessary cause of invasive cervical cancer worldwide. J Pathol 1999;189:12-19.

53. Meijer CJ, Berkhof J, Castle PE, et al. Guidelines for human papillomavirus DNA test requirements for primary cervical cancer screening in women 30 years and older. Int J Cancer 2009;124: 516-520.

54. Cuzick J, Cadman L, Mesher D, et al. Comparing the performance of six human papillomavirus tests in a screening population. $\mathrm{Br} \mathrm{J}$ Cancer 2013;108:908-913.

55. Nolte FS, Ribeiro-Nesbitt DG. Comparison of the Aptima and Cervista tests for detection of high-risk human papillomavirus in cervical cytology specimens. Am J Clin Pathol 2014;142:561-566.

56. Sauter JL, Mount SL, St John TL, et al. Testing of integrated human papillomavirus mRNA decreases colposcopy referrals: Could a change in human papillomavirus detection methodology lead to more cost-effective patient care? Acta Cytol 2014;58:162-166.

57. Knoepp SM, Kuebler DL, Wilbur DC. Correlation between hybrid capture II high-risk human papillomavirus DNA test chemiluminescence intensity from cervical samples with follow-up histologic results: A cytologic/histologic review of 367 cases. Cancer Cytopathol 2010;118:209-217.

58. Gibson JS. Nucleic acid-based assays for the detection of high-risk human papillomavirus: A technical review. Cancer Cytopathol 2014;122:639-645.

59. Solomon D, Schiffman M, Tarone R. Comparison of three management strategies for patients with atypical squamous cells of undetermined significance: Baseline results from a randomized trial. J Natl Cancer Inst 2001;93:293-299.

60. McMenamin M, McKenna M. Stability of human papillomavirus (HPV) in cervical ThinPrep specimens previously lysed with glacial acetic acid: Effect on cobas $4800 \mathrm{HPV}$ test performance. Cancer Cytopathol 2014;122:250-256.

61. Wright TCJ, Stoler MH, Behrens CM, et al. The ATHENA human papillomavirus study: Design, methods, and baseline results. Am J Obstet Gynecol 2012;206:46.e1-46.e11.

62. Krishna NB, LaBundy JL, Saripalli S, et al. Diagnostic value of EUS-FNA in patients suspected of having pancreatic cancer with a 


\section{CANBERK ET AL.}

focal lesion on CT scan/MRI but without obstructive jaundice. Pancreas 2009;38:625-630.

63. Wright TC, Stoler MH, Behrens CM, et al. Primary cervical cancer screening with human papillomavirus: End of study results from the ATHENA study using HPV as the first-line screening test. Gynecol Oncol 2015;136:189-197.

64. Castanon A, Landy R, Sasieni P. How much could primary human papillomavirus testing reduce cervical cancer incidence and morbidity? J Med Screen 2013;20:99-103.

65. de Kok IM, van Rosmalen J, Dillner J, et al. Primary screening for human papillomavirus compared with cytology screening for cervical cancer in European settings: Cost effectiveness analysis based on a Dutch microsimulation model. Bmj 2012;344:e670.

66. Lillsunde Larsson G, Carlsson J, Karlsson MG, et al. Evaluation of HPV genotyping assays for archival clinical samples. J Mol Diagn 2015;17:293-301.

67. Roncaglia MT, Tacla M, Vieira da Motta E, et al. Evaluation of the combination of cytology and hybrid capture to safely predict the highgrade lesion status of patients treated with conization with large loop excision of the transformation zone. Acta Cytol 2011;55:421-425.
68. Budal EB, Haugland HK, Skar R, et al. HPV DNA testing improves $\mathrm{CIN} 2+$ risk stratification and detection of $\mathrm{CIN} 2+$ in delayed triage of ASCUS and LSIL. A population-based follow-up study from Western Norway. Cancer Med 2014;3:182-189.

69. Verhoef VM, van Kemenade FJ, Rozendaal L, et al. Follow-up of high-risk HPV positive women by combined cytology and bimarker CADM1/MAL methylation analysis on cervical scrapes. Gynecol Oncol 2015;137:55-59.

70. Kawaguchi S, Shigehara K, Sasagawa T, et al. Liquid-based urine cytology as a tool for detection of human papillomavirus, Mycoplasma spp., and Ureaplasma spp. in men. J Clin Microbiol 2012; 50:401-406.

71. Aslan DL, Pambuccian SE, Prekker FL, et al. Accuracy of herpes simplex virus detection in liquid-based (SurePath) Papanicolaou tests: A comparison with polymerase chain reaction. Diagn Cytopathol 2008;36:94-103.

72. Sekhon HS, Press RD, Schmidt WA, et al. Identification of cytomegalovirus in a liquid-based gynecologic sample using morphology, immunohistochemistry, and DNA real-time PCR detection. Diagn Cytopathol 2004;30:411-417. 